INPLASY

PROTOCOL

To cite: Chen et al. Tuina combined with diet and exercise for simple obesity:A protocol for systematic review. Inplasy protocol 202210079. doi:

10.37766/inplasy2022.1.0079

Received: 16 January 2022

Published: 16 January 2022

Corresponding author: Xingquan Wu

wuxingquan2005@163.com

Author Affiliation:

Affiliated Hospital of Changchun University of

Chinese Medicine, Changchun, China.

\section{Support: Education}

Department of Jilin.

Review Stage at time of this submission: Preliminary searches.

Conflicts of interest: None declared.

\section{Tuina combined with diet and exercise for simple obesity: A protocol for systematic review}

Chen, L1; Cong, D2; Wang, G3; Sun, J4; Ji, Y5; Zhong, Z6; Liu, T7; Liu, J8; Chu, Y9; Wu, $X^{10}$.

Review question / Objective: Participants - Patients who were diagnosed with simple obesity, without limitation of age, gender, or racial. Interventions - The treatment schedule of the experimental group included Tuina combined with diet and exercise. Comparisons - The control group will receive diet and exercise therapy alone. Outcomes - The primary outcome will be total effective rate,BMI,BW,WC. Body mass index (BMI), which combines height and weight to determine obesity, is the most important indicator for the diagnosis and assessment of obesity. WHO defines obesity as the $B M I \geq 28$ $\mathrm{kg} / \mathrm{m} 2$ in Asian people and $\geq 30 \mathrm{~kg} / \mathrm{m} 2$ in non-Asian people. Waist circumference (WC): WC $\geq 90 \mathrm{~cm}$ for male and $80 \mathrm{~cm}$ for female. And then the age and gender obesity-related indicators of the included population are evaluated for statistical analysis, course of disease, education background, quality of life questionnaire score, self-satisfaction score.Secondary outcome measures: antipyretic time, cough duration, rhombus disappearance time, imaging transition time, serum C-reactive protein level (CRP) after treatment. Safety was referred to the incidence of adverse events (bleeding, pain, hematoma, syncope, etc.).

INPLASY registration number: This protocol was registered with the International Platform of Registered Systematic Review and Meta-Analysis Protocols (INPLASY) on 16 January 2022 and was last updated on 16 January 2022 (registration number INPLASY202210079).

\section{INTRODUCTION}

Review question / Objective: Participants Patients who were diagnosed with simple obesity, without limitation of age, gender, or racial. Interventions - The treatment schedule of the experimental group included Tuina combined with diet and exercise. Comparisons - The control group will receive diet and exercise therapy alone. Outcomes - The primary outcome will be total effective rate,BMI,BW,WC. Body mass index (BMI), which combines height and weight to determine obesity, is the most 
important indicator for the diagnosis and assessment of obesity. WHO defines obesity as the $B M I \geq 28 \mathrm{~kg} / \mathrm{m} 2$ in Asian people and $\geq 30 \mathrm{~kg} / \mathrm{m} 2$ in non-Asian people. Waist circumference (WC): WC $\geq 90 \mathrm{~cm}$ for male and $80 \mathrm{~cm}$ for female. And then the age and gender obesity-related indicators of the included population are evaluated for statistical analysis, course of disease, education background, quality of life questionnaire score, self-satisfaction score.Secondary outcome measures: antipyretic time, cough duration, rhombus disappearance time, imaging transition time, serum C-reactive protein level (CRP) after treatment. Safety was referred to the incidence of adverse events (bleeding, pain, hematoma, syncope, etc.).

Condition being studied: Improve the research plan.

\section{METHODS}

Participant or population: Patients diagnosed with simple obesity, an age between 18 and 60 , will be included in this study. There is no limitation on the sex, ethnicity, cultural background, and territory. Secondary obesity and gestational obesity will be excluded.

Intervention: The treatment schedule of the experimental group included Tuina combined with diet and exercise.

Comparator: The control group will receive diet and exercise therapy alone.

Study designs to be included: 1. Selection criteria1.1. Types of studies.We will include the randomized controlled clinical trials in this study. Published reports on the efficacy and clinical trials of Tuina combined with diet and exercise in the treatment of simple obesity are also included. RCTs that involve at least 1 Tuina combined with diet and exercise related treatment to simple obesity, and 1 control treatment will be included. The studies involving nonrandomized controlled trials (RCTs), animal experiments, reviews, and case series will be excluded.1.2. Types of patients. Patients diagnosed with simple obesi.
Eligibility criteria: RCT. Tuina combined with diet and exercise for simple obesity. Patients diagnosed with simple obesity , an age between 18 and 60 , will be included in this study. There is no limitation on the sex, ethnicity, cultural background, and territory. Secondary obesity and gestational obesity will be excluded.

Information sources: In order to evaluate the efficacy of Tuina combined with diet and exercise in the treatment of simple obesity, We will search PubMed, EMBASE, MEDLINE, Web of Science, Cochrane Library, WanFang Data, CBM, CNKI and VIP databases. We will collect randomized controlled trials published from inception to December 2021, regardless of language or form. The databases will be searched by combining the subject words with random words.

Main outcome(s): The primary outcome will be total effective rate,BMI,BW,WC. Body mass index (BMI), which combines height and weight to determine obesity, is the most important indicator for the diagnosis and assessment of obesity. WHO defines obesity as the $B M I \geq 28 \mathrm{~kg} / \mathrm{m} 2$ in Asian people and $\geq 30 \mathrm{~kg} / \mathrm{m} 2$ in non-Asian people. Waist circumference (WC): WC $\geq 90 \mathrm{~cm}$ for male and $80 \mathrm{~cm}$ for female. WC is the main index of abdominal obesity.

Additional outcome(s): And then the age and gender obesity-related indicators of the included population are evaluated for statistical analysis, course of disease, quality of life questionnaire score, selfsatisfaction score.

Quality assessment / Risk of bias analysis: Two researchers will evaluate the quality of the included studies under the guidance of the Cochrane Collaboration tool (RevMan 5.3) for RCTs.The 2 reviewers will complete this work independently. If there is any disagreement, they can discuss and resolve it, and the third researcher can be involved when necessary. The Cochrane Collaboration tool assesses the following seven domains: generation and allocation of random sequences, blinding of participants and personnel, blinding of 
outcome assessor, incomplete outcome data, selective reporting and other risk biases. According to the above evaluation items contained in the literature, we will designate the "unclear risk of bias" or "low risk of bias" or "high risk of bias" to provide a better understanding of the included study quality.

Strategy of data synthesis: When metaanalysis is permitted, statistical analysis will be performed using the Cochrane Review Manager (RevMan 5.3) software. Binary data represent risk ratios (RR), and continuous data represent the average difference between different trials when results are measured in the same way (MD). A 95\% confidence interval (Cl) will be used as the effective size for the joint analysis.

Subgroup analysis: Analysis of subgroups. If the condition allows, we will perform a subgroup analysis. The following subgroup analyses will be considered. 1.Gender of the patients. 2.Process of the patients. 3.Different types of Tuina therapies.

Sensitivity analysis: When sufficient data are available, sensitivity analysis will be performed to test the robustness of the primary outcomes, which includes assessing the quality of the methods, the quality of the studies, and the impact of sample size and missing data.

Country(ies) involved: China.

Keywords: Tuina; diet and exercise; simple obesity; protocol; systematic review.

Contributions of each author:

Author 1 - Lili Chen - conceptualization and formal analysis and methodology and writing- original draf.

Email: 1724435713@qq.com

Author 2 - Deyu Cong - conceptualization and formal analysis and methodology and writing- review \& editing.

Email: congdeyu666@sina.com

Author 3 - Gaofeng Wang - Formal analysis and writing- original draft.

Email: qswfer2008@163.com
Author 4 - Jiabao Sun - Methodology and writing- original draft.

Email: 444153695@qq.com

Author 5 - Yuanyuan Ji - Data curation.

Email: 2211842943@qq.com

Author 6 - Zhen Zhong - Data curation.

Email: 1248674961@qq.com

Author 7 - Tong Liu - Software.

Email: tongknow06@163.com

Author 8 - Jiayi Liu - Software.

Email: 954040542@qq.com

Author 9 - Yunjie Chu - Data curation and software.

Email: cyj2009@sina.cn

Author 10 - Xingquan $W u$ conceptualization and formal analysis and methodology and writing- review \& editing.

Email: wuxingquan2005@163.com 